\title{
Gene repressive mechanisms in the mouse brain involved in memory formation
}

\author{
Nam-Kyung Yu \& Bong-Kiun Kaang ${ }^{*}$ \\ Department of Biological Sciences, College of Natural Sciences, Seoul National University, Seoul 08826, Korea
}

\begin{abstract}
Gene regulation in the brain is essential for long-term plasticity and memory formation. Despite this established notion, the quantitative translational map in the brain during memory formation has not been reported. To systematically probe the changes in protein synthesis during memory formation, our recent study exploited ribosome profiling using the mouse hippocampal tissues at multiple time points after a learning event. Analysis of the resulting database revealed novel types of gene regulation after learning. First, the translation of a group of genes was rapidly suppressed without change in mRNA levels. At later time points, the expression of another group of genes was downregulated through reduction in mRNA levels. This reduction was predicted to be downstream of inhibition of ESR1 (Estrogen Receptor 1) signaling. Overexpressing Nrsn1, one of the genes whose translation was suppressed, or activating ESR1 by injecting an agonist interfered with memory formation, suggesting the functional importance of these findings. Moreover, the translation of genes encoding the translational machineries was found to be suppressed, among other genes in the mouse hippocampus. Together, this unbiased approach has revealed previously unidentified characteristics of gene regulation in the brain and highlighted the importance of repressive controls. [BMB Reports 2016; 49(4): 199-200]
\end{abstract}

Long-term memory formation involves dynamic gene regulatory events in the brain (Alberini and Kandel (2014) Cold Spring Harbor perspectives in biology 7:a021741, doi:10.1101/ cshperspect.a021741). In particular, de novo protein synthesis

*Corresponding author. E-mail: kaang@snu.ac.kr

http://dx.doi.org/10.5483/BMBRep.2016.49.4.044

Received 3 March 2016

Keywords: Contextual fear conditioning, Memory, Nrsn1, Ribosome profiling

Abbreviations: DEG, differentially expressed genes; ESR1, estrogen receptor 1; Nrsn1, Neurensin 1; RPF, ribosome-protected fragment

Perspective to: Cho et al (2015), Multiple repressive mechanisms in the hippocampus during memory formation, Science, 350(6256): 82-7, doi: $10.1126 /$ science.aac7368 during certain time windows (within $1 \mathrm{~h}$ or around $4 \mathrm{~h}$ ) after learning is known to be important for memory stabilization (Bourtchouladze et al. (1998) Learning \& Memory 5:365-374, doi:10.1101/lm.5.4.365). Despite this well-established notion, the identity of the proteins being synthesized during memory formation has not been systematically studied due to the lack of appropriate techniques until recently. Especially, genes that are regulated specifically at the translation step could not be identified by transcriptomic approaches. Moreover, the relative contribution of transcription and translation to regulation of each gene after learning remained largely unknown. To address these issues, we employed a technique called ribosome profiling (Brar and Weissman (2015) Nat Rev Mol Cell Biol 16:651-664, doi:10.1038/nrm4069). This method can quantify the translation rates of genes at the genomic scale by deep sequencing of the mRNA fragments protected by the ribosomes. Combined with RNA sequencing (RNA-seq) using the same sample, the translation efficiencies of individual genes can be calculated by normalizing the ribosome-protected fragment (RPF) read counts by the mRNA levels. By using this technique, our recent study mapped the temporal profile of translatomes and transcriptomes in the mouse brain after a learning event.

For the learning paradigm, we used contextual fear conditioning, in which subject mice were placed into a novel chamber and received a brief electric foot-shock after $2.5 \mathrm{~min}$ of exploration. In this paradigm, a single training leads to the formation of a long-lasting context-associated fear memory. Because hippocampal gene regulation and plasticity are known to be important for contextual fear conditioning, we obtained the mouse hippocampal samples at time points (5, 10 , and $30 \mathrm{~min}$ and $4 \mathrm{~h}$ ) when protein synthesis has been reported to be critical for memory stabilization. The samples were then subjected to ribosome profiling in parallel with RNA-seq. From three independent sets of experiments, we determined differentially expressed genes (DEG) at each time point by comparing the RPF values with those from the control group that did not undergo the conditioning.

Our unbiased approach revealed previously unidentified types of gene regulation during memory formation. At early time points after learning, we expected to uncover novel proteins that are rapidly synthesized from preexisting mRNA before the transcriptional changes occur. As expected, we 
found many DEGs at 5 and 10 min that showed alterations in RPF rather than in RNA levels, indicating that they were translationally regulated. Intriguingly, however, we found a remarkable reduction of RPF levels in 20 genes at these early time points, suggesting a rapid translational repression, which had not been reported before. Nrsn1, one of these early repressed genes, encodes the vesicular membrane protein believed to be involved in membrane trafficking and neurite outgrowth (Suzuki et al. (2007) Neuroscience Letters 421:152157 doi:10.1016/j.neulet.2007.03.077); however, yet this gene has not been studied in learning and memory. We found that the Nrsn1 protein level was also downregulated upon contextual fear conditioning in the hippocampus or upon neuronal activation in cultured neurons. Moreover, in our behavioral analyses, mice overexpressing Nrsn1 in the hippocampal neurons showed impaired long-term memory in contextual fear conditioning and object location task, suggesting the functional importance of the repression of Nrsn1.

At later time points of $30 \mathrm{~min}$ and $4 \mathrm{~h}$, we observed another type of downregulation in a group of genes that was predicted to be positively controlled by estrogen receptor 1 (ESR1). In contrast to the early translational repression, this later suppression appeared to be regulated through the amount of mRNA. To assess the functional importance of the inhibition of ESR1 signaling, we injected the ESR1 agonist, 1,3,5-tris(4hydroxyphenyl)-4-propyl-1H-pyrazole (PPT), into the mouse hippocampus after learning and found that mice with increased ESR1 activation showed weaker memory than control mice.

Moreover, we found that the translation efficiency of genes encoding translational machineries is suppressed in the mouse hippocampus. Accordingly, the ribosomal protein level and polyribosome fraction appeared to be lower in the hippocampus compared to other tissues. As protein synthesis is important for various brain functions including long-term memory formation, the tight regulation of translational machineries in the brain may have a functional impact on normal brain functions, which remains to be studied in the future.

Collectively, our results unveiled novel types of repressive gene regulation that may contribute to long-term memory formation. These findings raise numerous unexplored issues, such as the upstream mechanisms of these regulation types as well as their molecular and cellular consequences during memory formation. In addition to these gene repressive events, our study identified other protein-coding genes or long noncoding RNAs that were differentially expressed after learning. Studies on these newly identified individual genes may open a new avenue for studying the molecular mechanisms of memory formation and neuroplasticity.

Our study had a limitation in terms of identifying the precise locations of the molecular events. Brain tissue is heterogeneous, as diverse cell types or classes are intermingled. Accumulating evidence has shown that the gene expression patterns in the brain cells are in fact far more heterogeneous than had been thought (Cembrowski et al. (2016) Neuron 89(2):351-368, doi:10.1016/j.neuron.2015.12.013). Gene regulation in response to a certain stimulus could also be diversified according to the types of cells or the connectivity between cells. Gene expression analyses in mixed cell populations, such as those in our study, measure the average gene expression levels across a large number of cells and cannot accurately attribute the molecular events to specific cell populations. For example, we do not know whether the detected molecular events occurred in the activated or inhibited cells after learning. In addition, many changes that occur in only a small number of cells may not be detected. Moreover, molecular events that are localized at the subcellular level do not seem to appear in our database and those of other studies (Khoutorsky et al. (2013) Neuron 78(2):298-311, doi:10.1016/ j.neuron.2013.02.025) using total cell lysates. To resolve these issues and clearly understand gene regulation during memory formation, the combination of ribosome profiling with cell type- or population-specific cell sorting or labeling methods may provide great advances in future studies.

\section{ACKNOWLEDGEMENTS}

This work was supported by the National Honor Scientist Program of Korea (2012R1A3A1050385). 